\title{
Crashworthiness Design of a Steel-Aluminum Hybrid Rail Using Multi-response Objective-Oriented Sequential Optimization
}

\author{
Jianguang Fang ${ }^{1,4}, \mathrm{Na} \mathrm{Qiu}^{2, *}$, Xiuzhe $\mathrm{An}^{2}$, Fenfen Xiong ${ }^{3}$ Guangyong $\mathrm{Sun}^{4}$, Qing $\mathrm{Li}^{4}$ \\ ${ }^{1}$ School of Civil and Environmental Engineering, University of Technology Sydney, Sydney, NSW \\ 2007, Australia \\ ${ }^{2}$ School of Automotive Studies, Tongji University, Shanghai, 201804, China \\ ${ }^{3}$ School of Aerospace Engineering, Beijing Institute of Technology, Beijing 100081, China \\ ${ }^{4}$ School of Aerospace, Mechanical and Mechatronic Engineering, The University of Sydney, Sydney, \\ NSW 2006, Australia
}

\begin{abstract}
Hybrid structures with different materials have aroused increasing interest for their lightweight potential and excellent performances. For example, steel-aluminum hybrid structures could take advantage of both the light weight of aluminum and the high strength of steel in engineering applications. This study explored the optimization design of steel-aluminum hybrid structures for the highly nonlinear impact scenario. A metamodel based multi-response objective-oriented sequential optimization was adopted, where Kriging models were updated with sequential points. It was indicated that the sequential sampling strategy was able to obtain a much higher local accuracy in the neighborhood of the optimum and thus to yield a better optimum, although it did lead to a worse global accuracy over the entire design space. Furthermore, it was observed that the steel-aluminum hybrid structure was capable of decreasing the peak force and simultaneously enhancing the energy absorption, compared to the conventional mono-material structures.
\end{abstract}

Keywords: Hybrid structure, Multiresponse objective-oriented sequential sampling, Crashworthiness optimization, Kriging

\footnotetext{
${ }^{* *}$ Corresponding Author: Tel: +86-21-6958 9982; Email: nnaqiu@163.com
} 


\section{Introduction}

In recent years, protective structures have aroused extensive attention for impact and blast loadings [1]. S-shaped thin-walled structures have been widely used as a front rail in the automotive engineering to absorb energy during frontal crashes, as depicted in Fig. 1. However, the requirements for load withstanding capacity at different areas are not uniform $[2,3]$. To address this issue, hybrid materials, such as steel-aluminum hybrid structure, were investigated to improve passenger safety and weight efficiency. In this regard, Zhou et al. [4] explored the crashworthiness and lightweight design of steel-aluminum structure and found that steel-aluminum hybrid structure could increase the total absorbed energy by $117.83 \%$ and reduce the peak force and total mass by $16 \%$ and $7.73 \%$, respectively, compared to the base mono material model. Hosseini-Tehrani and Nikahd [5] pointed out that the steel-aluminum S-shaped rail could absorb more energy, produce a lower peak force and offer a lighter weight than the mono material counterpart. However, these above-mentioned studies are limited to the analysis of the crushing performance of the hybrid structure. In practical application, it needs to be further explored how to design the thicknesses and usage ratios of individual materials to excavate the potential of a hybrid structure.

Fig. $1 \mathrm{~S}$-shaped front rail in automotive engineering

Due to high nonlinearity of the crashworthiness problem, direct coupling the simulation model with an optimization algorithm is rather time-consuming or even prohibited in practice. As an alternative, the surrogate modeling or metamodeling technique is widely adopted in crashworthiness optimization [6]. In this regard, Qi et al. [7] combined the response surface metamodel with multi-objective optimization to improve the energy absorption of thin-walled rails under an oblique impact loading. Khakhali et al. [8] conducted a robust optimization design to maximize the energyabsorbing capacity for S-shaped box beams using polynomial metamodels. Fang et al. 
[9] conducted the multiobjective optimization of the functionally graded foam-filled tube under the lateral load based upon the multiobjective particle optimization (MOPSO) algorithm and Kriging modeling technique. Xiao et al. [10] investigated the crashworthiness of a novel functionally graded foam-filled bumper beam by utilizing the Kriging model. Qiu et al. [11, 12] proposed different multi-cell hexagonal tubes and compared their crashworthiness performance by employing Kriging surrogate model. Gao et al. [13] optimized the energy absorption capacity of foam-filled double ellipse tubes based on the Kriging model. Yamazaki and Han [14] aimed to maximize the crushing energy absorption of cylindrical shells based upon the response surface approximation technique. Hou et al. [15] conducted the crashworthiness optimization of corrugated beam guardrail based on RBF-MQ surrogate model. Wang et al. [16] developed a metamodeling optimization system and used to improve the crash behavior of the tube structure. Sun et al. optimized the crashworthiness of a novel criss-cross tubes based on the radial basic function surrogate model[17]. Fang et al. [18]comprehensively reviewed different surrogate models used in the crashworthiness optimization problem.

While the surrogate modeling is widely used in crashworthiness optimization, it is generally known that the accuracy of the metamodels largely depends on the number of training points [19]. Thus, it becomes a key issue to gain a satisfactory accuracy using the minimum number of sample points. Conventional one-stage sampling is used to capture the global trend of the real response with less flexibility, as the sampling points cannot be changed during the optimization. As a result, the one-stage sampling will not be able to guarantee a good local accuracy especially at the neighborhood region of the optimum. On the other hand, a more flexible alternative, which is referred to as sequential sampling strategy, can be utilized to refine the previous surrogate model with adding new sample points in an iterative fashion during the optimization [20]. Among various sequential sampling approaches, the objective-oriented sampling is a variant tailored for design optimization which takes the objective design into consideration [21-23]. However, in most of the previous works, objective-oriented sampling only deals with one response which combines objective and constraints. 
While Chen et al. [24] proposed an effective multi-response and multi-constraint metamodeling technique and introduced uncertainty quantification to take into account the confidence interval because of insufficient samples. Since a series of Boolean operation was utilized, this approach could deal with subspace with arbitrary shapes. In this paper, this sequential sampling strategy is utilized to optimize the steel-aluminum hybrid side rail structure.

This paper is structured as follows. Section 2 introduces the finite element modeling technique for both base model and hybrid steel-aluminum structure and described the optimization problem for the hybrid structure. Section 3 provides the detailed information about the multiresponse objective-oriented sequential sampling method. Section 4 discusses the optimization results and the effect of sequential sampling technique. Finally, the conclusions are drawn in Section 5.

\section{Crashworthiness of a hybrid side rail}

\subsection{Finite element modeling and experimental validation}

The structure studied herein is a thin-walled S-shaped front rail with a rectangle cross-section subjected to an axial impact loading (see in Fig. 2). The model was developed by using explicit non-linear finite element software LS-DYNA. The Belytschko-Tsay reduced integration shell elements with five integration points through thickness were adopted to model the tube. Stiffness-based hourglass control was used to avoid spurious zero energy deformation modes and reduced integration was utilized to avoid volumetric locking. "Automatic single surface" contact was selected to the thin walls themselves to avoid interpenetration of tube folding.

Fig. 2 Shape and cross section for S-shaped structure (thickness=1.3 mm)

The baseline design was made of mild steel, with the following properties: density $=7800 \mathrm{~kg} / \mathrm{m} 3$, Young's modulus $=206 \mathrm{Gpa}$, Poisson's ratio $=0.3$. The S-shaped tube was modeled by material model 24 in LS-DYNA, having a piecewise linear elastic- 
plastic behavior with strain hardening. To accurately describe the hardening characteristic in the FE model, the relationship between the plastic strain and plastic stress are shown as in Fig. 3 [25].

Fig. 3 Strain hardening data for mild steel for base model

As pointed out by Pan et al.[26] and Zhao et al. [27], the localized effect of welding properties on the global crashworthiness could be neglected, and thus the welding connection between two parts can be modeled with coincident nodes. The moving end of the tube was connected to the rigid body with the keyword *CONSTRAINED_EXTRA_NODES. The constant velocity of $2000 \mathrm{~mm} / \mathrm{s}$ was adopted to consider the low-velocity impact as in reference [4]. For the low-velocity impact, strain rate effect of the materials was considered throughout the optimization process below. The load was applied at the center of gravity of this rigid body. The rear end of the tube was fully clamped. The boundary condition applied to the hybrid structure is shown in Fig. 4.

In order to determine the size of elements, a convergence test was conducted to minimize the effect of mesh refinement on the accuracy of the numerical results in terms of the energy absorption and the maximum force. It was found that $5 \mathrm{~mm}$ was the optimal mesh size of the tube, as it could reduce computing time without sacrificing the simulation accuracy too much.

Fig. 4 The boundary condition applied to the hybrid structure

\subsection{Experimental validation}

The quasi-static experimental data was used to validate the FE modeling approach, since experimental data for the low-velocity impact is not available. Specifically, the experimental test was conducted with the cross-head velocity of $5 \mathrm{~mm} / \mathrm{min}$ [28]. Therefore, a load velocity of $0.5 \mathrm{~mm} / \mathrm{s}$ with a smooth ramp [28] was used to simulate the quasi-static load condition for the purpose of experimental validation. Also, the 
Fig. 5 Experimental [29] and numerical results for global deformation

Fig. 6 Comparison of energy-displacement responses between the numerical and experimental results

\subsection{Hybrid steel-aluminum S-shaped side rail structure}

For the hybrid steel-aluminum structure, the high-strength steel DP300/500 was adopted as the base steel material, with the following mechanical properties: density $=7800 \mathrm{~kg} / \mathrm{m}^{3}$, Poisson's ratio $=0.3$, Young's modulus $=206 \mathrm{Gpa}$, and the relationship between stress and strain rate (Fig. 7) was also considered. Aluminum alloy AA6060T4 was employed as the front-end material. Its mechanical properties is described as follows: density $=2700 \mathrm{~kg} / \mathrm{m}^{3}$, Poisson's ratio $=0.3$, Young's modulus $=68.2 \mathrm{Gpa}$, and initial yielding stress $=80 \mathrm{Mpa}[30]$. The relationship between the true stress and plastic strain is shown in Fig. 8, which was defined to accurately describe the hardening effect in FE model. Since the aluminum is insensitive to the strain rate, the rate-dependent effect was not measured.

Fig. 7 Relationship between plastic strain and true stress at various strain rates for DP300/500

Fig. 8 Strain hardening data for AA6060T4 


\subsection{Formulation of the optimization problem}

To systematically study and optimize the crashworthiness of the S-shaped front rail structure, it is essential to determine the crashworthiness criteria before the optimization process. There have been many different criteria which are available to evaluate the crashing characteristics of different structures. Energy absorption (EA) is widely used to estimate the energy absorption capability of absorbers as follows

$$
E A(d)=\int_{0}^{d} F(x) \mathrm{d} x
$$

where $F(x)$ is the crashing force in the axial direction and $x$ is the crash displacement. Obviously, a higher $E A$ value indicates a higher capability of energy absorption. In our case, when the displacement reaches $200 \mathrm{~mm}$ the front rail has experienced sufficient deformation (in particularly two plastic hinges have formed at a much earlier time). After $200 \mathrm{~mm}$, the reaction force remains at a low level and less energy will be absorbed. In other words, further deformation could provide limited benefits of energy absorption. Instead, it could lead to severe intrusion to occupant compartment, which is prohibited in real-life applications. Therefore, $E A$ during the first $200 \mathrm{~mm}$ was adopted as a performance indicator.

In automotive applications, a large impact force often leads to a high acceleration and severe injury or even death of occupants. Therefore, the peak impact force $F_{\max }$ represents a critical indicator to the occupant survival rate when an impact occurs. In this study, the objective was to maximize $E A$ while constraining the peak force and the total mass less than the predefined levels $\left(F_{\max }^{*}\right.$ and $M^{*}$ respectively). Besides, the rear part of the hybrid rail was high-strength steel for offering the required stiffness and preventing severe intrusion to the occupant compartment in practical applications, while the front part was aluminum to lower the peak force. The length ratio of the aluminum part to the whole length $\left(\lambda=L_{1} / L\right)$, the aluminum thickness $\left(t_{A L}\right)$ and the steel thickness $\left(t_{A H H S}\right)$ were taken as the design variables (Fig. 9). Thus, this optimization problem could be expressed mathematically as follows: 
Fig. 9 Design variables

$$
\begin{aligned}
& \text { Find } \quad \mathbf{x}=\left(\lambda, t_{A L}, t_{A H H S}\right)^{T} \\
& \left\{\begin{array}{c}
\text { Maximize } \quad f(\mathbf{x})=E A\left(\lambda, t_{A L}, t_{A H H S}\right) \\
\text { s.t. }\left\{\begin{array}{c}
g_{1}(\mathbf{x})=F_{\max }^{*}-F_{\max }\left(\lambda, t_{A L}, t_{A H H S}\right)>0 \\
g_{2}(\mathbf{x})=M^{*}-M\left(\lambda, t_{A L}, t_{A H H S}\right)>0 \\
0.1 \leq \lambda \leq 0.25 \\
2.0 \mathrm{~mm} \leq t_{A L} \leq 4.0 \mathrm{~mm} \\
1.0 \mathrm{~mm} \leq t_{A H H S} \leq 1.5 \mathrm{~mm}
\end{array}\right.
\end{array}\right.
\end{aligned}
$$

To improve the computational efficiency, the metamodeling or surrogate modeling technique was adopted. The surrogate model can provide an approximate functional relation between design variables and specific responses with a moderate number of computational analyses [31]. Therefore, after generating the training points, various metamodels, namely Polynomial response surface (PRS), moving least square (MLS), Kriging (KRG) and radial basis function (RBF) can be implemented to evaluate the performance responses. The Kriging model is chosen herein mainly because the following two reasons: (1) it allows better capturing nonlinear response with respect to usage of the two materials and (2) the predicted error of its estimated response value can be easily obtained as a by-product that will form a basis of sequential sampling strategy to be outlined below.

The Kriging model was originally developed for mining and geostatistical applications involving spatially and temporally correlated data [32]. The Kriging model assumes the deterministic response of a system to be a stochastic process function $y(\mathbf{x})$, consisting of a regression model and a stochastic error [33]:

$$
y(\mathbf{x})=\mathbf{f}(\mathbf{x})^{\mathrm{T}} \boldsymbol{\beta}+z(\mathbf{x})
$$

where $\boldsymbol{\beta}$ is the column vector of regression parameters, $\boldsymbol{\beta}=\left[\beta_{1}, \beta_{2}, \ldots, \beta_{p}\right]^{\mathrm{T}} ; \mathbf{f}(\mathbf{x})$ is the column vector of basis functions, $\mathbf{f}(\mathbf{x})=\left[f_{1}(\mathbf{x}), f_{2}(\mathbf{x}), \ldots, f_{p}(\mathbf{x})\right]^{\mathrm{T}} ; p$ denotes the number of basis functions; $z(\mathbf{x})$ represents a stochastic parameter with zero mean, variance $\sigma^{2}$, and nonzero covariance. The covariance matrix of $z(\mathbf{x})$ is given as:

$$
\operatorname{Cov}\left[z\left(\mathbf{x}_{i}\right), z\left(\mathbf{x}_{j}\right)\right]=\sigma^{2} \mathbf{R}\left[R\left(\mathbf{x}_{i}, \mathbf{x}_{j}\right)\right]
$$


where $\mathbf{R}$ is a correlation matrix defined by Gaussian correlation function $R\left(\mathbf{x}_{i}, \mathbf{x}_{j}\right)$ as follows:

$$
R\left(\mathbf{x}_{i}, \mathbf{x}_{j}\right)=\exp \left[-\sum_{k=1}^{N} \theta_{k}\left|x_{i, k}-x_{j, k}\right|^{2}\right]
$$

where $\theta_{k}$ is the unknown correlation parameter used to fit the model.

Then, the predicted estimate $\hat{y}(\mathbf{x})$ of response $y(\mathbf{x})$ is given as:

$$
\hat{y}(\mathbf{x})=\mathbf{f}(\mathbf{x})^{\mathrm{T}} \hat{\boldsymbol{\beta}}+\mathbf{r}^{\mathrm{T}}(\mathbf{x}) \mathbf{R}^{-1}\left(\mathbf{y}_{s}-\mathbf{F} \hat{\boldsymbol{\beta}}\right)
$$

where $\mathbf{y}_{s}=\left[y\left(\mathbf{x}_{1}\right), y\left(\mathbf{x}_{2}\right), \ldots, y\left(\mathbf{x}_{n_{s}}\right)\right]^{\mathrm{T}}$ is the response vector of the $n_{s}$ training points $\mathbf{x}_{s}=\left\{\mathbf{x}_{1}, \mathbf{x}_{2}, \ldots, \mathbf{x}_{n_{s}}\right\}$ which are obtained from the finite element analyses, and $\mathbf{F}=\left[\mathbf{f}\left(\mathbf{x}_{1}\right), \mathbf{f}\left(\mathbf{x}_{2}\right), \ldots, \mathbf{f}\left(\mathbf{x}_{n_{s}}\right)\right]^{\mathbf{T}} \quad$ is $\quad$ a $\quad n_{s} \times p$ matrix. $\mathbf{r}^{\mathrm{T}}(\mathbf{x})=\left[R\left(\mathbf{x}, \mathbf{x}_{1}\right), R\left(\mathbf{x}, \mathbf{x}_{2}\right), \ldots, R\left(\mathbf{x}, \mathbf{x}_{n_{s}}\right)\right]^{\mathrm{T}}$ is a correction vector that implies how close between training points and untried points is. $\hat{\boldsymbol{\beta}}$ is the general least square estimator given as follows:

$$
\hat{\boldsymbol{\beta}}=\left(\mathbf{F}^{\mathrm{T}} \mathbf{R}^{-1} \mathbf{F}\right)^{-1} \mathbf{F}^{\mathrm{T}} \mathbf{R}^{-1} \mathbf{y}_{s}
$$

The estimate to the variance of training data from the global model is described as

$$
\hat{\sigma}^{2}=\frac{\left(\mathbf{y}_{s}-\mathbf{F} \hat{\boldsymbol{\beta}}\right)^{\mathrm{T}} \mathbf{R}^{-1}\left(\mathbf{y}_{s}-\mathbf{F} \hat{\boldsymbol{\beta}}\right)}{n_{s}}
$$

For calculating $\theta_{k}$ in Eq. (5), the maximum likelihood estimates can be used by solving the following the maximization problem over the interval $\theta_{k}>0$, as

$$
\max \left(-\frac{n_{s} \ln \left(\hat{\sigma}^{2}\right)+\ln |\mathbf{R}|}{2}\right)
$$

where both $\hat{\sigma}^{2}$ and $|\mathbf{R}|$ are the functions of $\theta_{k}$.

Kriging model provides estimation to the prediction error from an unobserved point, which is also called as the mean squared error (MSE):

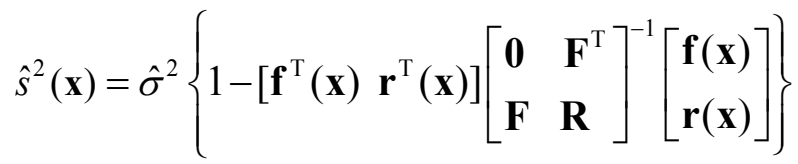

In this study, we adopt the ordinary Kriging model [34], in which the regression 


\section{Multi-Response Objective-Oriented Sequential Sampling procedure}

Several previous works [35-42] have shown certain advantages provided by a particular sequential sampling strategy. In this study, an objective-oriented sampling strategy addressed in the literature [24] was applied to the crashing performance optimization. At the initial iteration, only a small number of sampling points were prepared, and then three new points selected in the feasible region were added to update the metamodels at each iteration.A flowchart of the proposed sequential sampling strategy for refining Kriging-based optimization is provided in Fig. 10.

Fig. 10 Flowchart of the sequential sampling strategy

Step1: The sampling points at the first iteration were considered to estimate the global trend of the responses. In this study, the optimal Latin Hypercube sampling (OLHS ) method was used to generate 15 initial sampling points.

Step2: Fit the Kriging metamodels $\hat{f}_{\text {obj }}(\mathbf{x})$ for the objective and $\hat{g}_{1}(\mathbf{x})$ and $\hat{g}_{2}(\mathbf{x})$ for the two constraints. The prediction error of the Kriging models from an unobserved point could be estimated by the mean squared error (MSE), which is related with the variance of training data and the position of predicted point. In this study, the sampling error was taken into consideration by calculating the $\operatorname{MSE}\left(\hat{\sigma}_{o b j}\right)$ for the objective and $\operatorname{MSE}\left(\hat{\sigma}_{g_{i}}\right)$ for the constraint metamodels.

$(1-\alpha) \%$ confidence level $(\mathrm{CI})$ for objective metamodel:

$$
\left[\hat{f}_{o b j}(x)-k \hat{\sigma}_{o j b}(x), \hat{f}_{o b j}(x)+k \hat{\sigma}_{o j b}(x)\right]
$$

As self-evident $(1-\alpha) \%$ confidence level (CI) for constraint metamodels:

$$
\left[\hat{g}_{i}(x)-k \hat{\sigma}_{g_{i}}(x), \hat{g}_{i}(x)+k \hat{\sigma}_{g_{i}}(x)\right]
$$


Here $k$ denotes the constant determined by the confidence level.

Step3: Determine the feasible regions and run optimizations for the best and worst cases using the artificial bee colony algorithm $[43,44]$. The optimal problem was defined in Eq. (13) and Eq. (14) for the best and worst case respectively, considering the confidence level.

$$
\begin{array}{ll}
\max & \hat{f}_{o b j}(x)-k \hat{\sigma}_{o b j}(x) \\
\text { s.t. } & \hat{g}_{1}+k \hat{\sigma}_{g 1} \geq 0 \\
& \hat{g}_{2}+k \hat{\sigma}_{g 2} \geq 0 \\
& \\
\max & \hat{f}_{o b j}(x)+k \hat{\sigma}_{o b j}(x) \\
\text { s.t. } & \hat{g}_{1}-k \hat{\sigma}_{g 1} \geq 0 \\
& \hat{g}_{2}-k \hat{\sigma}_{g 2} \geq 0
\end{array}
$$

Step4: Check the result convergence by comparing the objective value between the best $\left(O_{B}\right)$ and worst $\left(O_{W}\right)$ cases. If the difference was smaller than $5 \%$, current optimal $x^{*}$ was updated as the final optimal point. If not, go to Step5.

Step5: Identify the regions of interest determined by the objective function $\phi_{o b j}(x)$. In the whole design space, the region where the objective solution was worse than the worst case $\left(\phi_{1}(x)=\hat{f}_{o b j}(x)+k \hat{\sigma}_{o b j}-O_{W}\right)$ or better than the best case ( $\phi_{2}(x)=-\left(\hat{f}_{o b j}(x)-k \hat{\sigma}_{o b j}-O_{B}\right)$ would be ruled out. $\phi_{o b j}(x)$ was calculated as

$$
\phi_{o b j}(x)=-\max \left(\phi_{1}(x), \phi_{2}(x)\right)
$$

Step6: Identify maximum feasible region $\left(\phi_{g}(x)\right)$ based on the two constraints considering quantified uncertainties,

$$
\phi_{g}(x)=\min \left(\hat{g}_{i}(x)+\hat{\sigma}_{g_{i}}(x)\right) i=1,2
$$

Step7: Synthesize the final interest region, which was the intersection of the regions determined in Step 5 and Step 6 by Eq. (17).This region was considered to be the new feasible region for determination the location of the new training points. The final interest region would shrink with the process of the level set sequential sampling method. 


\subsection{Optimization results}

In this paper, the optimal problem has three responses, i.e. one objective and two constraints. The metamodels were constructed based on 15 initial samples, which uniformly spread over the whole design space, using Latin Hypercube sampling (OLHS ) method. The sequential sampling strategy was conducted by adding three new training points at each stage. Finally, the solution was considered to be converged after five sequential sampling stages, and the final optimum is presented in Table 1, compared with the results of the baseline model. In addition, the crashworthiness of mono high-strength steel was also optimized based on 20 OLHS points and polynomial response surface models. As shown in Table 1, the pure high-strength steel performed the worst in peak force and energy absorption due to the high strength of the material. The Fig. 11 plots the deformation mode of the baseline model and the optimal hybrid model, respectively. Obviously, the optimal solution of the hybrid structure had a much smaller maximum force than the base model, which indicates that the optimal hybrid structure was more likely to increase the occupant survival rate. In addition, the energy absorption of the hybrid structure was increased by $11.4 \%$ with the $7 \%$ mass reduction than the baseline model. 
Table 1 Comparison between the optimal hybrid model and the base model

In brief, the optimal steel-aluminum hybrid structure generated from the sequential sampling strategy showed a better performance in terms of crashworthiness and weight efficiency.
(a) baseline model
(b) optimal hybrid model

Fig. 11 Deformation mode

Fig. 12 Comparison of the force impact force between the optimal hybrid model and the base model

As the aluminum at the front end of the hybrid model was less rigid than the mild steel of the base model, the hybrid model experienced a lower peak force than the mild steel model (see Fig. 12). Moreover, due to the lightweight effect of aluminum, the high-strength steel part of the hybrid structure could be much thicker than that of baseline model (see Table 2). As the bending hinge occurred in the high strength steel part, the hybrid model could provide more resistance to the bending deformation as shown in Fig. 12, offering more energy absorption. Overall, the hybrid steel-aluminum structure could enhance the energy absorption while maintaining the peak force and mass at an allowable level).

\subsection{Effect of sequential technique}

Fig. 13 shows the history of the sequential sampling strategy, where the squares denote the previous samples, the diamond represents the optimum at the current stage and the circles stand for the selected training points for next iteration. The boundary surfaces of region of interested obtained by Eq. (17) are displayed by the blue surfaces. It could be seen that the region of interest, i.e., the space between the two blue surfaces, shrank gradually toward the final optimum as the sequential sampling strategy progresses with three points added per iteration. Obviously, the region of interest always embodied the optimum throughout the whole optimization process. It was also 
observed that the sample points located in the neighborhood of the optimum became denser. As a result, the local accuracy at this region was improved with the sequential strategy, which could help attain the optimum design.

Fig. 13 Sampling region of the sequential strategy. (squares: previous points; circles: newly selected points; diamonds: optimal solution)

To show its effectiveness, the result of the sequential sampling strategy was compared with that of the one-stage strategy with the same total number of sampling points, as shown in Table 2 .

Table 2 Optimization results obtained from the two sampling strategies

To evaluate the accuracy of the metamodels, additional 5 checking points spreading over the whole design region was generated using the OLHS method.

Two commonly-used metrics, i.e. average absolute error $e_{\text {avg }}$ relative maximum absolute error $e_{\max }$, as formulated in Eqs. (19) and (20) respectively, were selected to assess the accuracy of the metamodels constructed by the sequential sampling and onestage strategies.

$$
\begin{aligned}
& e_{\text {avg }}=\frac{1}{d} \sum_{i=1}^{d}\left[\frac{\left|y_{i}-\bar{y}_{i}\right|}{\left|y_{i}\right|}\right] \\
& e_{\max }=\max _{i \in d}\left[\frac{\left|y_{i}-\bar{y}_{i}\right|}{\left|y_{i}\right|}\right]
\end{aligned}
$$

As shown in Table 3, the one-stage sampling strategy seemed to perform better in the global accuracy than the sequential sampling strategy. However, the optimum generated by the sequential sampling strategy was much better than the one-stage solution and yielded more accurate results by comparing the predicted and real responses. Although the optimization based on the one-stage sampling strategy had a higher accuracy over the entire design domain, it failed to find the true optimum. On the other hand, the sequential sampling strategy generated many points in the neighborhood of the optimum. As a result, the local accuracy was greatly improved, which was much more critical for the optimization. 
Table 3 Accuracy assessment of metamodels in the entire design space

In the local region of $0.22 \leq \lambda \leq 0.25, \quad 2.6 \mathrm{~mm} \leq t_{A L} \leq 2.9 \mathrm{~mm}$ and

$1.2 \mathrm{~mm} \leq t_{A H H S} \leq 1.3 \mathrm{~mm}$, additional 5 examination training points were generated by the OLHS method. As shown in Table 4, the sequential sampling strategy was demonstrate to achieve a much higher local accuracy than the one-stage sampling strategy. Because, in this case, the sequential sampling strategy successfully generated more sampling points in the neighborhood of the optimal solution, which helps to improve the local accuracy of the established metamodels and seek the true optimal design.

Table 4 Accuracy assessment of the metamodels in the neighborhood of the optimum

\section{Concluding remarks}

To obtain the optimal thicknesses and usage ratios for each individual material in a steel-aluminum hybrid S-shaped front side rail, a metamodel-based optimization was developed, which integrating with the multi-response objective-oriented sequential sampling strategy. The optimization problem was defined to maximize the $E A$ (energy absorption) with the constraints of mass and peak force. To improve the optimization efficiency, the sequential sampling strategy was used to update the Kriging metamodel adaptively by adding certain training points at each iteration.

Through a comparative study of the sequential sampling and one-stage sampling strategies, it was found that although the one-stage sampling method achieved more accurate metamodels over the whole design domain, it may obtain a false optimum due to the lower local accuracy. On the other hand, the sequential sampling strategy generated more training points in the neighborhood of the optimum, thereby achieving higher local accuracy and obtaining the true optimum successfully. After 5 iterations of sequential sampling with a total of 30 training points, the optimal thicknesses and usage ratios for the hybrid structure was identified. Compared with the base model, $t$ the steelaluminum hybrid structure increased the energy absorption while constraining the peak force and total weight at a lower level. 


\section{Acknowledgment}

This work was supported by The National Natural Science Foundation of China (51575399).

\section{References:}

[1] Fan Z, Lu G, Liu K. Quasi-static axial compression of thin-walled tubes with different cross-sectional shapes. Engineering Structures. 2013;55:80-9.

[2] Shi Y, Zhu P, Shen L, Lin Z. Lightweight design of automotive front side rails with TWB concept. ThinWalled Structures. 2007;45:8-14.

[3] Fang J, Gao Y, Sun G, Xu C, Li Q. Multiobjective sequential optimization for a vehicle door using hybrid materials tailor-welded structure. Proceedings of the Institution of Mechanical Engineers, Part C: Journal of Mechanical Engineering Science. 2015;230:3092-100.

[4] Zhou Y, Lan F, Chen J. Crashworthiness research on S-shaped front rails made of steel-aluminum hybrid materials. Thin-Walled Structures. 2011;49:291-7.

[5] Hosseini-Tehrani P, Nikahd M. Two materials S-frame representation for improving crashworthiness and lightening. Thin-Walled Structures. 2006;44:407-14.

[6] Fang J, Gao Y, Sun G, Qiu N, Li Q. On design of multi-cell tubes under axial and oblique impact loads. Thin-Walled Structures. 2015;95:115-26.

[7] Qi C, Yang S, Dong F. Crushing analysis and multiobjective crashworthiness optimization of tapered square tubes under oblique impact loading. Thin-Walled Structures. 2012;59:103-19.

[8] Khakhali A, Nariman-Zadeh N, Darvizeh A, Masoumi A, Notghi B. Reliability-based robust multiobjective crashworthiness optimisation of S-shaped box beams with parametric uncertainties. International Journal of Crashworthiness. 2010;15:443-56.

[9] Fang J, Gao Y, Sun G, Zhang Y, Li Q. Parametric analysis and multiobjective optimization for functionally graded foam-filled thin-wall tube under lateral impact. Computational Materials Science. 2014;90:265-75.

[10] Xiao Z, Fang J, Sun G, Li Q. Crashworthiness design for functionally graded foam-filled bumper beam. Advances in Engineering Software. 2015;85:81-95.

[11] Qiu N, Gao Y, Fang J, Feng Z, Sun G, Li Q. Theoretical prediction and optimization of multi-cell hexagonal tubes under axial crashing. Thin-Walled Structures. 2016;102:111-21.

[12] Qiu N, Gao Y, Fang J, Feng Z, Sun G, Li Q. Crashworthiness analysis and design of multi-cell hexagonal columns under multiple loading cases. Finite Elements in Analysis and Design. 2015;104:89101.

[13] Gao Q, Wang L, Wang Y, Guo F, Zhang Z. Optimization of foam-filled double ellipse tubes under multiple loading cases. Advances in Engineering Software. 2016;99:27-35.

[14] Yamazaki K, Han J. Maximization of the crushing energy absorption of cylindrical shells. Advances in Engineering Software. 2000;31:425-34.

[15] Hou S, Tan W, Zheng Y, Han X, Li Q. Optimization design of corrugated beam guardrail based on RBF-MQ surrogate model and collision safety consideration. Advances in Engineering Software. 
2014;78:28-40

[16] Wang H, Li E, Li GY, Zhong ZH. Development of metamodeling based optimization system for high nonlinear engineering problems. Advances in Engineering Software. 2008;39:629-45.

[17] Sun G, Pang T, Fang J, Li G, Li Q. Parameterization of criss-cross configurations for multiobjective crashworthiness optimization. International Journal of Mechanical Sciences. 2017;124-125:145-57.

[18] Fang J, Sun G, Qiu N, Kim NH, Li Q. On design optimization for structural crashworthiness and its state of the art. Structural and Multidisciplinary Optimization. 2016:1-29.

[19] Fang J, Gao Y, Sun G, Zhang Y, Li Q. Crashworthiness design of foam-filled bitubal structures with uncertainty. International Journal of Non-Linear Mechanics. 2014;67:120-32.

[20] Fang J, Gao Y, Sun G, Li Q. Development of a novel identification platform for automotive dampers. International Journal of Vehicle Design. 2014;66:272.

[21] Cox DD, John S. A statistical method for global optimization. Systems, Man and Cybernetics, 1992, IEEE International Conference on: IEEE; 1992. p. 1241-6.

[22] Jones DR. A taxonomy of global optimization methods based on response surfaces. Journal of global optimization. 2001;21:345-83.

[23] Sekishiro M, Venter G, Balabanov V. Combined kriging and gradient-based optimization method. 11th AIAA/ISSMO Multidisciplinary Analysis and Optimization Conference2006. p. 6-8.

[24] Chen S, Xiong Y, Chen W. Multiresponse and Multistage Metamodeling Approach for Design Optimization. AIAA Journal. 2009;47:206-18.

[25] Consortium U-A. ULSAB-AVC Technical Transfer Dispatch\# 6-Body Structure Materials. May; 2001.

[26] Pan F, Zhu P, Zhang Y. Metamodel-based lightweight design of B-pillar with TWB structure via support vector regression. Computers \& Structures. 2010;88:36-44.

[27] Zhao KM, Chun BK, Lee JK. Finite element analysis of tailor-welded blanks. Finite Elements in Analysis and Design. 2001;37:117-30.

[28] Khalkhali A, Masoumi A, Darvizeh A, Jafari M, Shiri A. Experimental and Numerical Investigation into the Quasi-Static Crushing Behaviour of the S-Shape Square Tubes. Journal of Mechanics. 2011;27:585-96.

[29] Khalkhali A, Masoumi A, Darvizeh A, Jafari M, Shiri A. Experimental and Numerical Investigation into the Quasi-Static Crushing Behaviour of the S-Shape Square Tubes. Journal of Mechanics. 2011;27:585-96.

[30] Santosa SP, Wierzbicki T, Hanssen AG, Langseth M. Experimental and numerical studies of foamfilled sections. International Journal of Impact Engineering. 2000;24:509-34.

[31] Fang J, Gao Y, Sun G, Li Q. Multiobjective reliability-based optimization for design of a vehicle door. Finite Elements in Analysis and Design. 2013;67:13-21.

[32] Sacks J, Welch WJ, Mitchell TJ, Wynn HP. Design and analysis of computer experiments. Statistical science. 1989;4:409-23.

[33] Gao Y, Sun F. Multi-disciplinary optimisation for front auto body based on multiple optimisation methods. International Journal of Vehicle Design. 2011;57:178-95.

[34] Sasena MJ. Flexibility and efficiency enhancements for constrained global design optimization with kriging approximations: Citeseer; 2002.

[35] Kitayama S, Arakawa M, Yamazaki K. Sequential approximate optimization using radial basis function network for engineering optimization. Optimization and Engineering. 2011;12:535-57.

[36] Bonte MH, Fourment L, Do T-t, Van den Boogaard A, Huetink J. Optimization of forging processes using finite element simulations. Structural and Multidisciplinary Optimization. 2010;42:797-810. 
[37] Booker AJ, Dennis Jr J, Frank PD, Serafini DB, Torczon V, Trosset MW. A rigorous framework for optimization of expensive functions by surrogates. Structural optimization. 1999;17:1-13.

[38] Huang D, Allen T, Notz W, Miller R. Sequential kriging optimization using multiple-fidelity evaluations. Structural and Multidisciplinary Optimization. 2006;32:369-82.

[39] Huang D, Allen TT, Notz WI, Zeng N. Global optimization of stochastic black-box systems via sequential kriging meta-models. Journal of global optimization. 2006;34:441-66.

[40] Jones DR, Schonlau M, Welch WJ. Efficient global optimization of expensive black-box functions. Journal of global optimization. 1998;13:455-92.

[41] Sasena MJ, Papalambros P, Goovaerts P. Exploration of metamodeling sampling criteria for constrained global optimization. Engineering optimization. 2002;34:263-78.

[42] Xiong F, Xiong Y, Chen W, Yang S. Optimizing Latin hypercube design for sequential sampling of computer experiments. Engineering optimization. 2009;41:793-810.

[43] Karaboga D, Basturk B. A powerful and efficient algorithm for numerical function optimization: artificial bee colony ( $A B C$ ) algorithm. Journal of global optimization. 2007;39:459-71.

[44] Karaboga D, Basturk B. On the performance of artificial bee colony (ABC) algorithm. Applied Soft Computing. 2008;8:687-97. 


\section{Table Captions}

Table 1 Comparison between the optimal hybrid model and the base

Table 2 Optimization results obtained from the two sampling strategies

Table 3 Accuracy assessment of metamodels in the entire design space

Table 4 Accuracy assessment of metamodels in the neighborhood of the optimum 


\section{Figure Captions}

Fig. 1 S-shaped front rail in automotive engineering

Fig. 2 Shape and cross section for s-shaped structure (thickness $=1.3 \mathrm{~mm}$ )

Fig. 3 Strain hardening data for mild steel

Fig. 4 The boundary condition applied to the hybrid structure

Fig. 5 Experimental [29] and numerical results for global deformation

Fig. 6 Comparison of the numerical and experimental energy-displacement responses

Fig. 7 Relationship between plastic strain and true stress at various strain rates for DP300/500

Fig. 8 Strain hardening data for AA6060T4

Fig. 9 Design variables

Fig. 10 Flowchart of the sequential sampling strategy

Fig. 11 Deformation mode

Fig. 12 Comparison of the force impact force between the optimal hybrid model and the base model

Fig. 13 Sampling region of the sequential strategy. (squares: previous points; circles: newly selected points; diamonds: optimal solution) 
Table 1 Comparison between the optimal hybrid model and the base model

\begin{tabular}{cccccccc}
\hline & $\lambda$ & $\begin{array}{c}t_{\text {base }} \\
(\mathrm{mm})\end{array}$ & $\begin{array}{c}t_{A H H S} \\
(\mathrm{~mm})\end{array}$ & $\begin{array}{c}t_{A L} \\
(\mathrm{~mm})\end{array}$ & $\begin{array}{c}F_{\max } \\
(\mathrm{kN})\end{array}$ & $\begin{array}{c}E A \\
(\mathrm{~J})\end{array}$ & $\begin{array}{c}M \\
(\mathrm{~kg})\end{array}$ \\
\hline Baseline model & $/$ & 1.3 & $/$ & $/$ & 28.271 & 1364.9 & 1.670 \\
Hybrid model & 0.2476 & $/$ & 2.8022 & 1.2781 & 26.174 & 1530 & 1.355 \\
Mono AHHS & $/$ & $/$ & 1.2138 & $/$ & 28.271 & 1247 & 1.559 \\
\hline
\end{tabular}


Table 2 Optimization results obtained from the two sampling strategies

\begin{tabular}{llllllll}
\hline & $\lambda$ & $t_{A H H S}(\mathrm{~mm})$ & $t_{A L}(\mathrm{~mm})$ & & $F_{\max }(\mathrm{kN})$ & $E A(\mathrm{~J})$ & $m(\mathrm{~kg})$ \\
\hline Sequential & 0.2476 & 2.8022 & \multirow{2}{*}{1.2781} & Predicted & 21.932 & 1552.4 & 1.547 \\
sampling & & & & Real & 21.949 & 1520.0 & 1.554 \\
& & & & Error & $0.14 \%$ & $2.08 \%$ & $0.45 \%$ \\
One-stage & 0.1170 & \multirow{2}{*}{2.6669} & \multirow{2}{*}{1.0894} & Predicted & 21.384 & 1568.2 & 1.533 \\
sampling & & & & Real & 18.517 & 1144.4 & 1.375 \\
& & & & Error & $13.4 \%$ & $27.02 \%$ & $10.31 \%$ \\
\hline
\end{tabular}


Table 3 Accuracy assessment of metamodels in the entire design space

\begin{tabular}{lllll}
\hline & metrics & $F_{\max }$ & $E A$ & $m$ \\
\hline Sequential sampling & $e_{\text {avg }}(\%)$ & 6.24 & 8.20 & 2.40 \\
& $e_{\max }(\%)$ & 11.58 & 16.60 & 7.93 \\
One-stage sampling & $e_{\text {avg }}(\%)$ & 3.08 & 2.76 & 2.76 \\
& $e_{\max }(\%)$ & 5.71 & 8.63 & 7.72 \\
\hline
\end{tabular}


Table 4 Accuracy assessment of metamodels in the neighborhood of the optimum

\begin{tabular}{ccccc}
\hline & metrics & $E A$ & $F_{\max }$ & $m$ \\
\hline \multirow{3}{*}{ Sequential sampling } & $e_{\text {avg }}(\%)$ & 0.72 & 2.85 & 1.09 \\
& $e_{\max }(\%)$ & 1.45 & 3.98 & 1.46 \\
One-stage sampling & $e_{\text {avg }}(\%)$ & 3.40 & 3.05 & 1.89 \\
& $e_{\max }(\%)$ & 5.45 & 4.67 & 2.91 \\
\hline
\end{tabular}




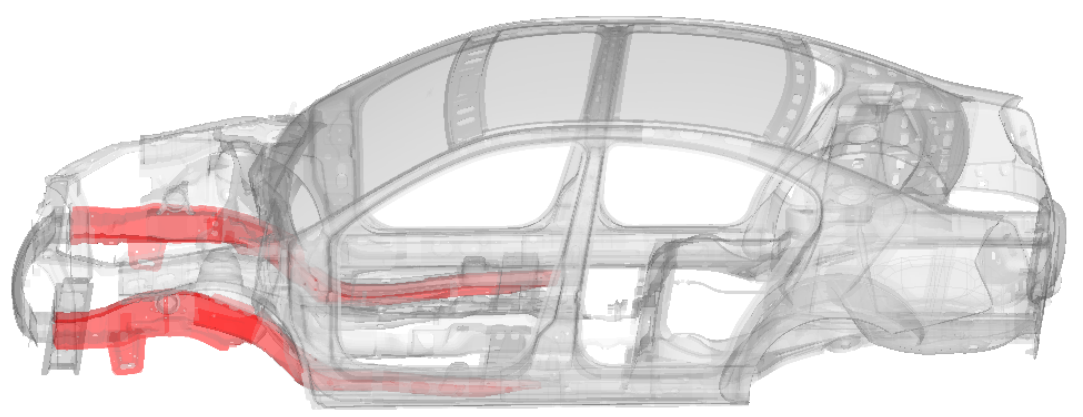

Fig. 1 S-shaped front rail in automotive engineering 


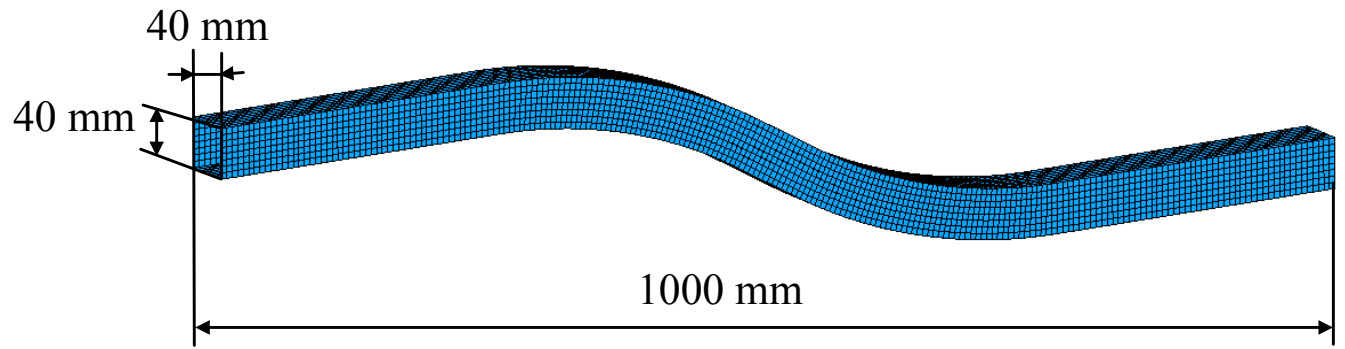

Fig. 2 Shape and cross section for s-shaped structure (thickness $=1.3 \mathrm{~mm}$ ) 


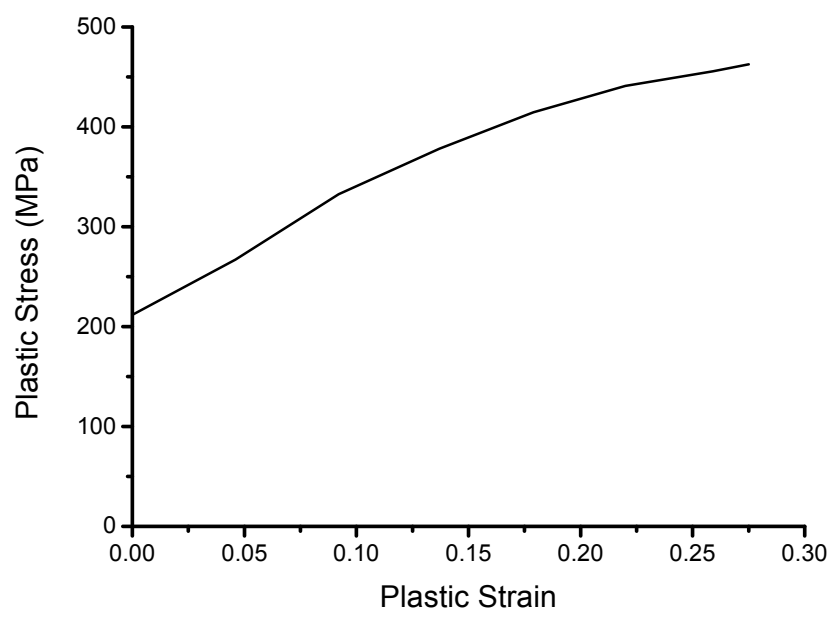

Fig. 3 Strain hardening data for mild steel for base model 


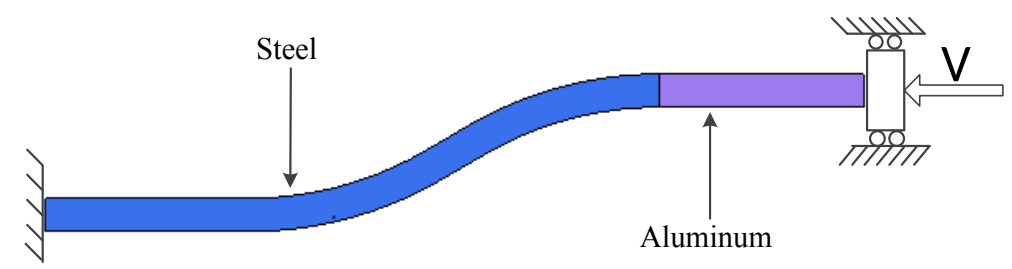

Fig. 4 Boundary condition applied to the hybrid structure 

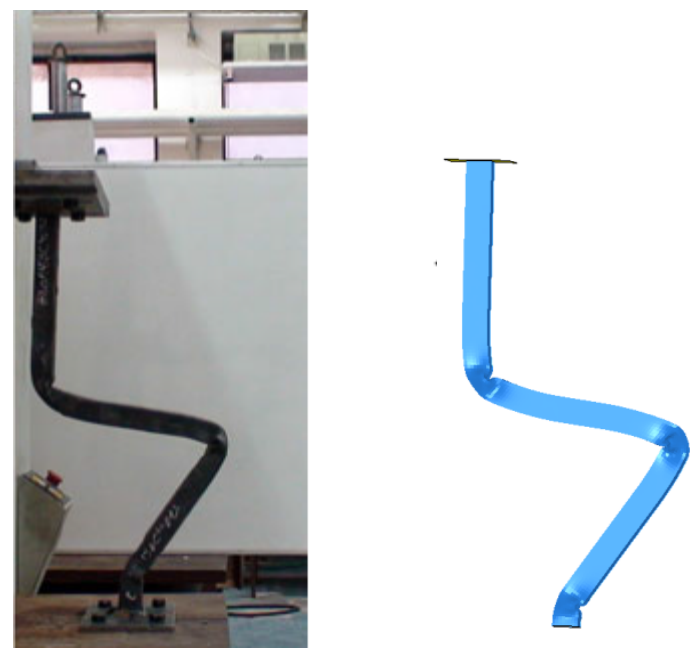

Fig. 5 Experimental [29] and numerical results for global deformation 


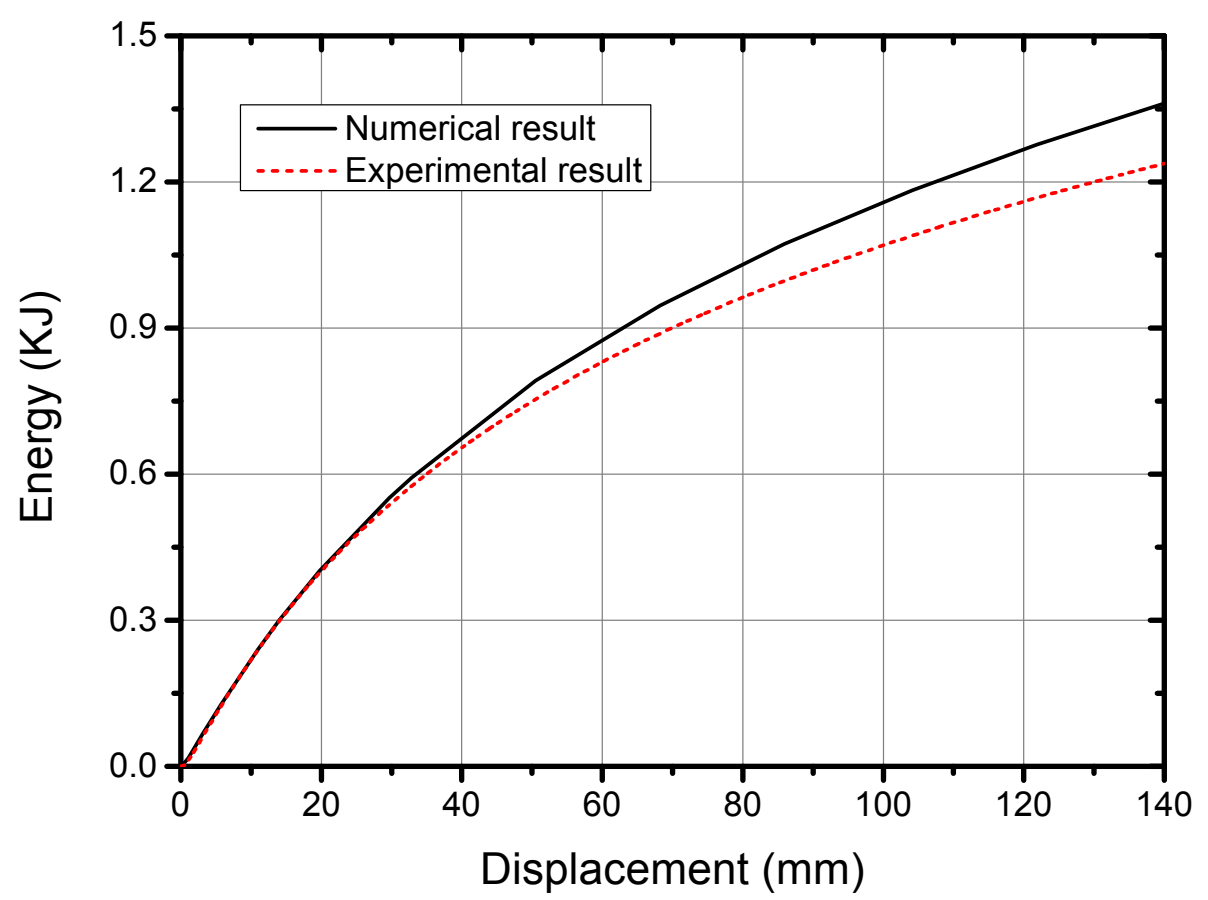

Fig. 6 Comparison of the numerical and experimental load-displacement responses 
Fig. 7 Relationship between plastic strain and true stress at various strain rates for DP300/500 


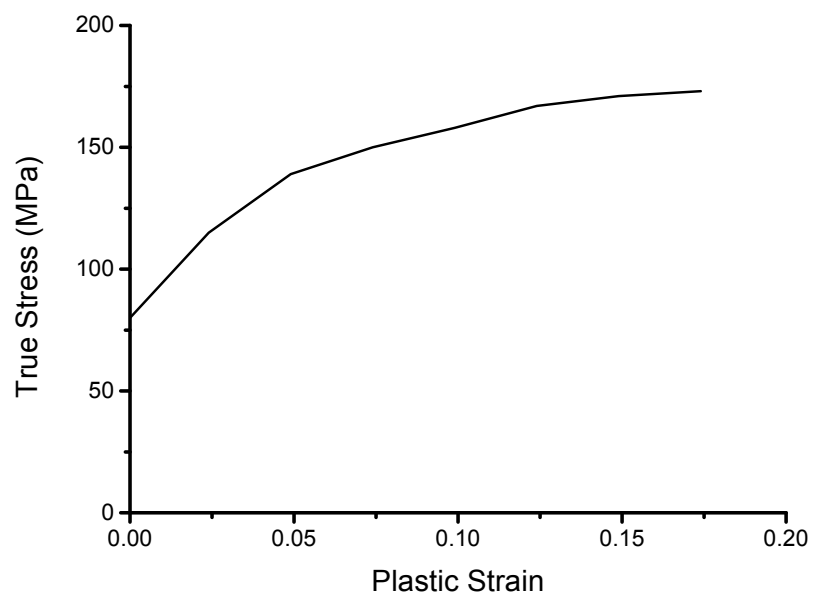

Fig. 8 Strain hardening data for AA6060T4 


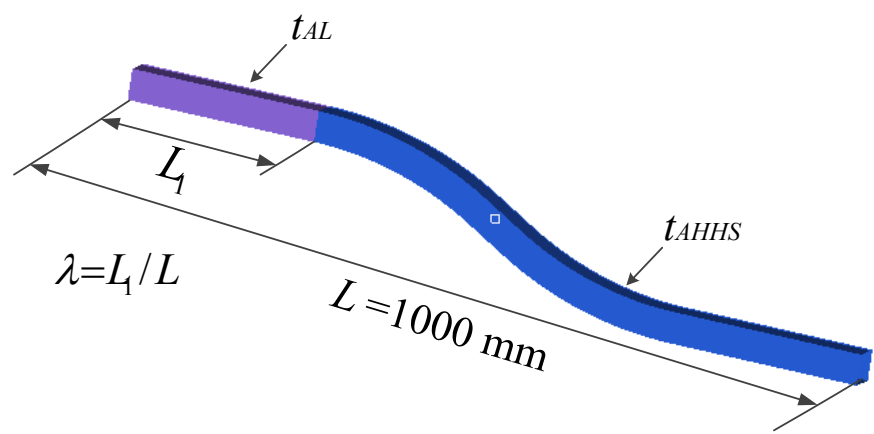

Fig. 9 Design variables 


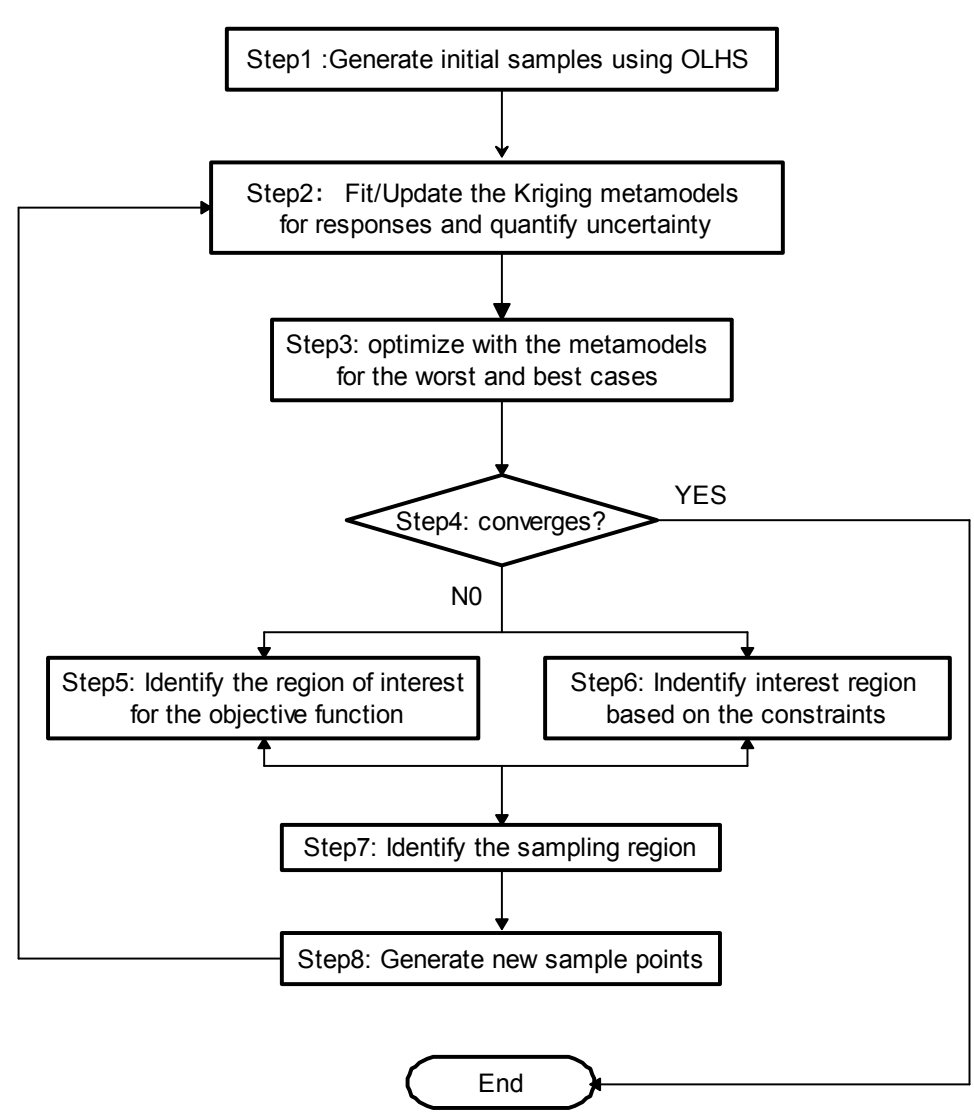

Fig. 10 Flowchart of the sequential sampling strategy 


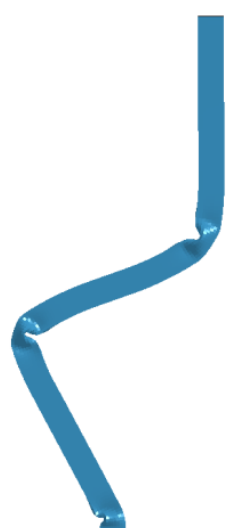

(a) baseline model

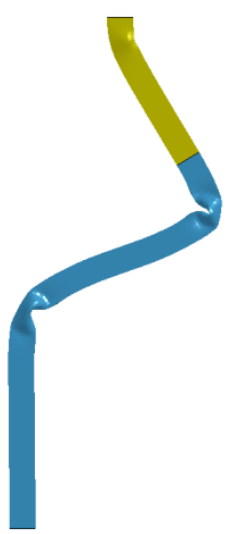

(b) optimal hybrid model

Fig. 11 deformation mode 


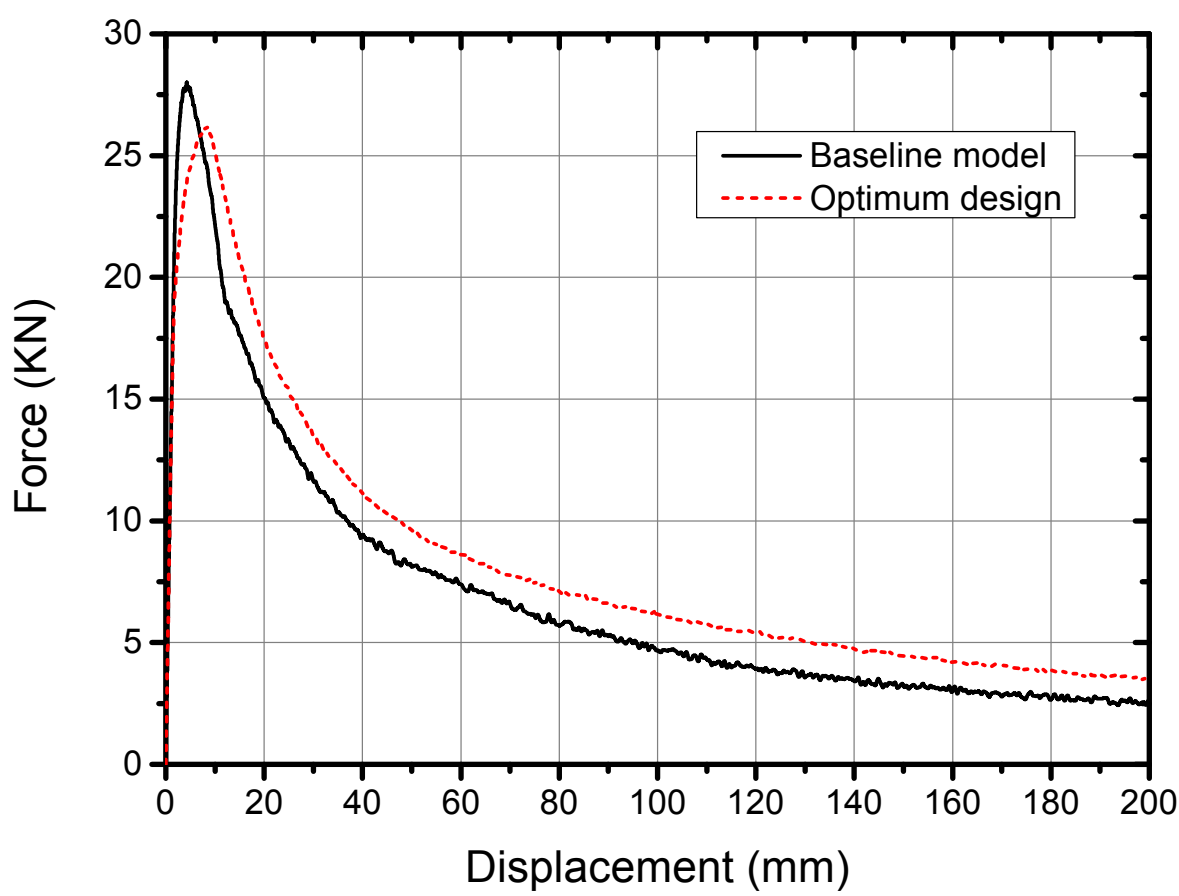

Fig. 12 Comparison of the force impact force between the optimal hybrid model and the base model 


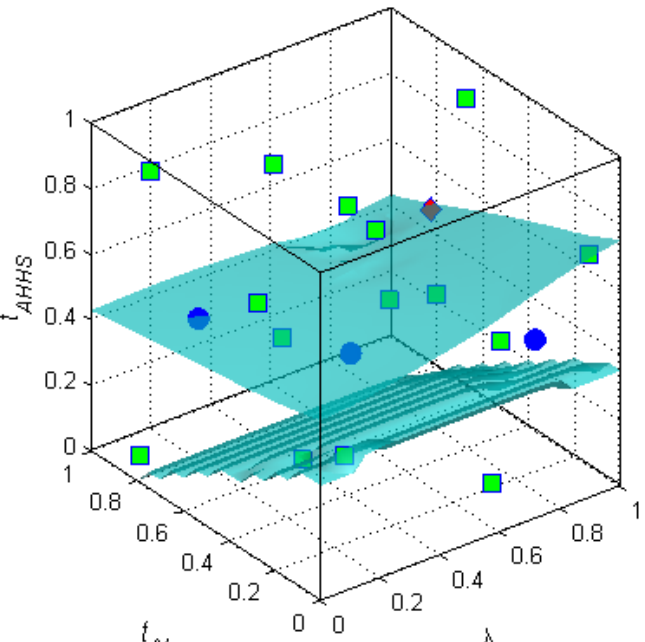

a) Sampling region of initial iteration

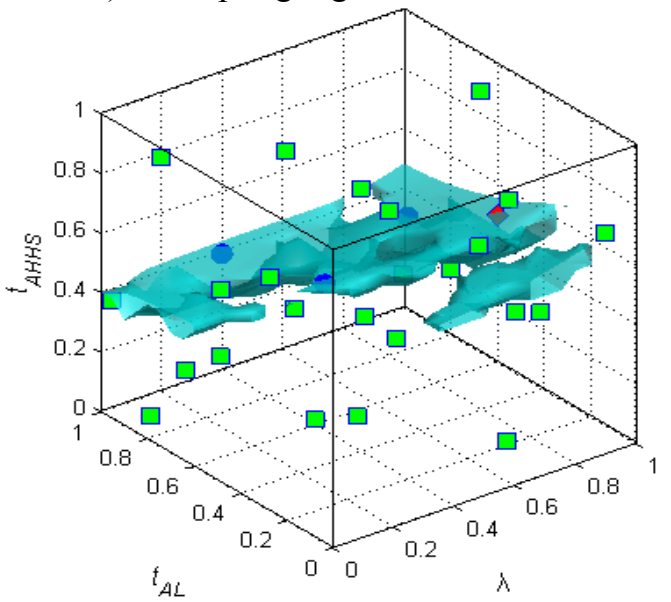

c) Sampling region of Iteration 3

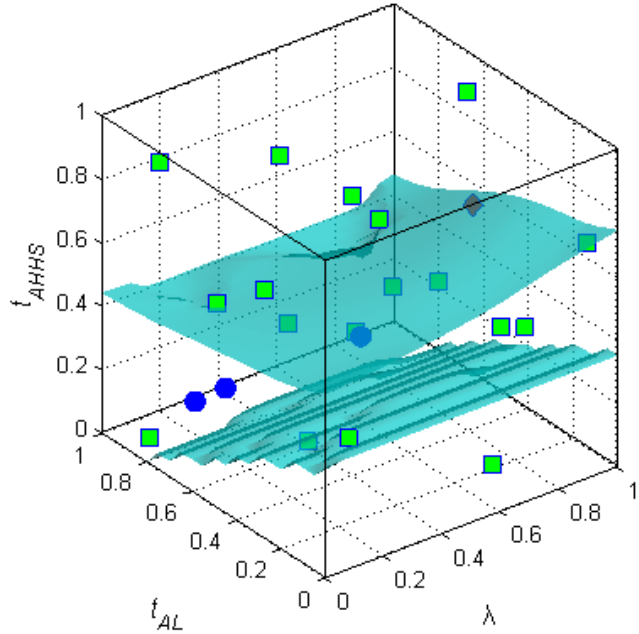

b) Sampling region of Iteration 1

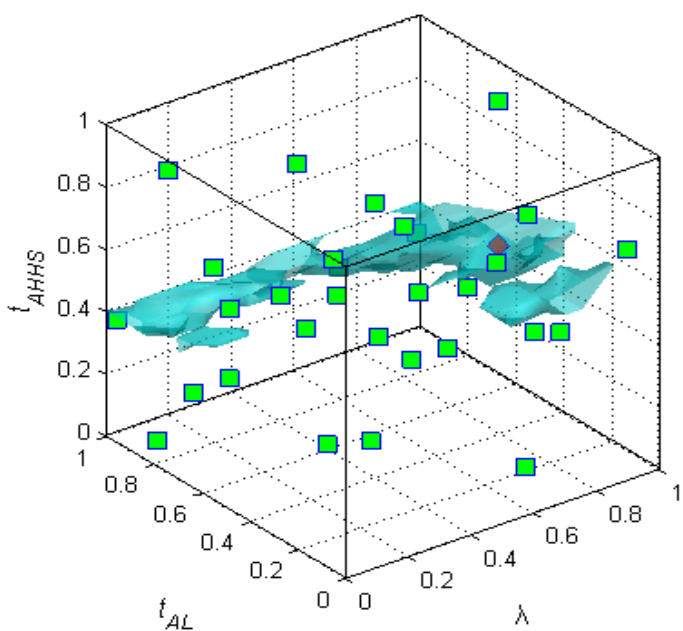

d) Sampling region of Iteration 5

Fig. 13 Sampling region of the sequential strategy. (squares: previous points; circles: newly selected points; diamonds: optimal solution) 


\section{Highlight}

1. Steel-Aluminum Hybrid structures were optimized to achieve better crashworthiness

2. Artificial bee colony $(\mathrm{ABC})$ algorithm was adopted to seek the optimum

3. A multi-response objective-oriented sequential sampling strategy was compared with one-stage sampling strategy 